\title{
Am Anfang steht das Unbehagen
}

Seit drei Jahrzehnten engagiert sich das Ehepaar Hess-Cabalzar für ein menschengerechtes Gesundheitswesen. Die Psychotherapeutin und der Arzt stossen Grundsatzdiskussionen an über das Menschenbild in der Medizin, über die Folgen der Ökonomisierung insbesondere am Patientenbett und zu Grenzen des Machbaren. Wichtig ist ihnen die Reintegration der Geisteswissenschaften und der Kunst in die medizinische Versorgung. Als Plattform für ihre Aktivitäten haben sie 2009 die «Akademie Menschenmedizin» gegründet.

Interview: Anna Sax

anna.sax[at]saez.ch

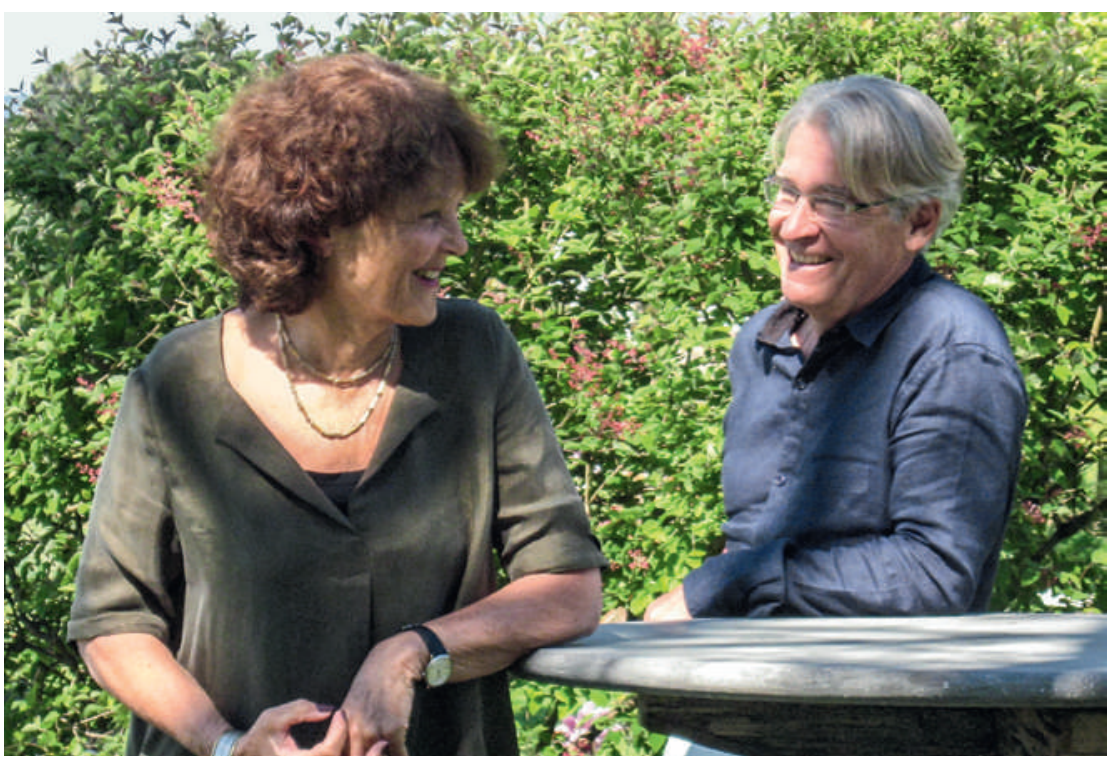

Annina Hess-Cabalzar und Christian Hess geht es um eine Reintegration der Geisteswissenschaften und der Kunst, der Medical Humanities, in die Medizin. ein wachsendes Unbehagen gegenüber den Entwicklungen in der Medizin, die sich immer stärker an Technologie und Machbarkeitsfantasien orientierte. Im Fokus unserer Arbeit steht aber der Mensch, und so bildete die Orientierung an einem expliziten
Menschenbild den Kern unserer Überlegungen. Ein integrales Menschenbild, wie wir es definiert haben, fordert die Reintegration der Geisteswissenschaften als besonders wichtigen Aspekt einer Neuorientierung in der Gesundheitsversorgung. Die Verknüpfung von Na-

\section{«Die Verknüpfung von Naturwissenschaft und Geisteswissenschaft war historisch eine Selbstverständlichkeit.»}

turwissenschaft und Geisteswissenschaft war historisch eine Selbstverständlichkeit. In den letzten 150 Jahren nahm die Naturwissenschaft jedoch, wohl dank ihrer eindrücklichen Erfolge, immer stärker Überhand, und die Einheit von materiellen und seelisch, geistigen Aspekten ist auseinandergebrochen. Nun geht es darum, das immer noch gültige Modell in einer modernen Form wieder zu beleben, im Sinne einer Reintegration der Geisteswissenschaften und der Kunst - der Medical Humanities - in die Medizin.

«Menschenmedizin» hat in manchen Ohren einen leicht esoterischen Beiklang - sind Sie esoterisch veranlagt? Annina Hess-Cabalzar (AHC): Ganz und gar nicht. Esoterik in ihrer ursprünglichen Bedeutung wäre eine Lehre, die nur einem begrenzten «inneren Kreis» zugänglich ist - eine Art «Geheimclub», der sich vom Bestehenden abgrenzt. Das entspricht überhaupt nicht unserer Idee. Wir bewegen uns innerhalb des Systems und innerhalb der Schulmedizin, und hier geht es um eine Stärkung der Beziehungsmedizin gegenüber der Apparatemedizin. In der Tat ist es so, dass die Alternativmedizin den Beziehungsaspekt aufgenommen hat und stark betont. Menschenmedizin ist jedoch kein alternativmedizinisches, sondern ein schulmedizinisches Konzept, anwendbar auf alle medizinischen Bereiche und 
Disziplinen, wobei die Beziehung und der Mensch im Fokus stehen.

$\mathrm{CH}$ : Ein schulmedizinisches Konzept, ergänzt durch Geisteswissenschaften ...

AHC: ... eigentlich völlig selbstverständlich und naheliegend.

Sie haben im Spital Affoltern während über zwei Jahrzehnten das "Modell Affoltern" aufgebaut und umgesetzt. Wie soll man sich das vorstellen?

\section{«Menschenmedizin ist jedoch kein alternativmedizinisches, sondern ein schulmedizinisches Konzept.»}

AHC: Das hat sich über die Jahre entwickelt. Zum Glück konnten wir lange Zeit in einem guten Umfeld arbeiten. So war es möglich, die Spitalleitung und die Mitarbeitenden für das Modell zu gewinnen, und es zeigte sich, dass sie sich gerne darauf einliessen. Zunächst einmal ging es darum, sich darüber klar zu werden, dass wir es im Spital mit kranken Menschen und nicht mit Fällen zu tun haben, dass wir Menschen und nicht Diagnosen behandeln und begleiten. Jede Krankengeschichte ist ein Unikat, jede Patientin, jeder Patient hat seine eigene Geschichte. Ein zentrales Element der Menschenmedizin ist die kunstgestützte Psychotherapie, die hilft, das Geschehen im Rahmen der Lebensgeschichte zu verstehen, hilft Hintergründe und Zusammenhänge wahrzunehmen und notwendige Veränderungen anzugehen. Dieses Angebot steht allen Patientinnen und Patienten unabhängig von der medizinischen Diagnose zur Verfügung.

Was machten Sie in Affoltern anders? Können Sie uns ein Beispiel geben?

$\mathrm{CH}$ : Exemplarisch ist die Geschichte eines Lehrlings, der mit dem Auto gegen einen Kandelaber fuhr und verletzt zu uns kam. Er stand kurz vor der Lehrabschlussprüfung. Dieses Beispiel zeigt auch den volkswirtschaftlichen Nutzen eines solchen Ansatzes. Wir behandelten seine Verletzungen ... und jetzt erzähl du weiter, Annina.

AHC: Wir merkten, dass hinter dem Unfall noch mehr steckt, also boten wir ihm eine Auseinandersetzung in der Psychotherapie an. Es zeigte sich in seinen in der Nacht gemalten Bildern rasch, dass es zu Hause massive Gewalt gab, und dass sein Unfall wohl auch eine verzweifelte Reaktion auf diesen Stress war, der durch die bevorstehende Prüfung noch verstärkt wurde. Wir konnten mit ihm und seinem Umfeld zusammen umgehend eine Veränderung der Wohnsituation herbeiführen, und schliesslich wurde er nicht nur gesund, sondern schaffte auch den Lehrabschluss. Insgesamt waren lediglich fünf Therapiestunden nötig. Die Ärzte hätten ihn auch einfach zusammenflicken und wieder nach Hause schicken können. Aber dann wäre er wieder der gleichen Situation ausgesetzt gewesen, die letztendlich zu seinem Unfall geführt hatte und hätte die Lehre wohl abgebrochen.

Wurde das Modell Affoltern auch an anderen Orten umgesetzt?

AHC: Mehrere Spitäler und Kliniken haben uns eingeladen, das Modell vorzustellen. Ich selbst konnte es am Inselspital in der Kardiologie einführen und etablieren. Auch die Maternité des Stadtspitals Triemli schaffte entsprechende Stellen. Aus den vielen Vortragsanfragen ergab sich dann auch die Idee, einen interdisziplinären Lehrgang zu entwickeln.

$\mathrm{CH}$ : Wichtig ist, dass sich das Modell nicht nur in einem Kleinspital umsetzen lässt. Es kann ebenso gut in der Hightech-Medizin, in der Rehabilitation, in einer Gruppenpraxis oder in einer einzelnen $\mathrm{Ab}$ teilung implementiert werden.

AHC: Es ist denkbar, dass unser interdisziplinäres Team eine Klinik oder Praxis besucht und einige Tage deren Alltag erlebt und beobachtet. Aufgrund dieser Erfahrungen werden Empfehlungen erarbeitet, wie die menschengerechte Medizin gestärkt werden kann. Analog zu der Kunst und zu den individuellen Behandlungen soll auch die Unternehmenskultur angepasst und einmalig gestaltet sein. Es gilt auch hier Originale zu erarbeiten, nicht Kopien.

Können Sie mir noch etwas mehr über die Kunst in Ihrem Konzept erzählen?

AHC: Dass man Kunst im Sinne eines Heilmittels integriert, ist aus der Menschheitsgeschichte heraus völlig naheliegend. Kunst gehört zum Menschsein. Sie hat immer schon Freud und Leid dargestellt, das Existenzielle der Menschen gespielt, formuliert oder gemalt. Ein Heilsystem kann ohne Kunst nicht auskommen. Es mag auf den ersten Blick erstaunen, dass wir sie in den Alltag der Schulmedizin eines öffentlichen Spitals mit Grundversorgung integrieren konnten, aber wir haben über zwanzig Jahre die Erfahrung gemacht, dass für Patienten das sinnliche Erleben $\mathrm{zu}$ einem Instrumentarium wird, um die Krankheit zu bewältigen und einen Umgang mit ihr $\mathrm{zu}$ finden. Das gilt für alle Altersgruppen und Bildungsschichten. Eine Krankheit kann das Leben völlig verändern. Kunst kann helfen, diese Veränderungen zu begreifen und letztendlich zu akzeptieren.

\section{Haben eigentlich alle Patientinnen und Patienten Zugang zur Kunst?}

AHC: Man glaubt oft, Kunst sei elitär. Der Kunstmarkt ist es vielleicht, nicht aber die Kunst. Sie betrifft jeden Menschen. Ich bin überzeugt, dass es keinen Menschen gibt, der nicht in irgendeiner Form einen Bezug hat zu Musik, Texten oder Farben. Diese Tatsache nutzbar zu machen für einen eigenen Erkenntnisprozess, darum geht es schliesslich in der 
kunstgestützten Psychotherapie. Es ist im Prinzip einfach: Es braucht nur den Schritt über die Schwelle ins Machen hinein, und plötzlich ist es möglich, einen Teil der Lebensgeschichte zu schreiben oder mit anderen Mitteln zu gestalten. Und dann ist es eben das Eigene und nicht das, was sonst jemand über einen gesagt hat. Wenn ich am Schluss meine Schlafstörungen mit Musik statt mit Temesta behandeln kann, dann leuchtet der Nutzen wohl ein.

\section{«Viele spüren das Unbehagen, doch sie funktionieren weiter passiv im System.»}

$\mathrm{CH}$ : Wenn Medizin mehr als nur Reparaturwerkstatt sein will, nämlich Heilkunst, dann ist sie darauf angewiesen, diese Bereiche einzubeziehen.

Sprechen wir noch über die Akademie Menschenmedizin. Diese haben Sie vor fünf Jahren gegründet - was möchten Sie damit erreichen?

$\mathrm{CH}$ : Die Entstehungsgeschichte der Akademie hat sich in mehreren Phasen abgespielt: Am Anfang stand das Unbehagen, über das wir zu Beginn gesprochen haben. Im Spital Affoltern entwickelten und praktizierten wir ein interdisziplinäres Betreuungsund Behandlungskonzept, welches Medizin, Geisteswissenschaften und Kunst integriert. Nach zehn Jahren Praxis verfassten wir unser Buch «Menschenmedizin», in dem wir das Menschenbild und die Vision eines Gesundheitssystems darlegten, welches Gesundsein und Kranksein in eine Balance zu bringen sucht. Schliesslich haben wir als weiteren Schritt die «Akademie Menschenmedizin» gegründet.

AHC: Im Rahmen der Akademie wollten wir eigentlich zunächst den vorhin erwähnten Lehrgang entwickeln ...

$\mathrm{CH}:$... doch dann kam die Politik dazwischen: Die neue Spitalfinanzierung über DRG wurde im Parlament beschlossen, und wir nahmen diese Entwicklung zum Anlass, eine Plattform gegen die zunehmende Ökonomisierung am Patientenbett aufzubauen. Zahlreiche Persönlichkeiten schlossen sich der Forderung nach einem DRG-Moratorium an. Die DRG wurden anfangs 2012 trotzdem umgesetzt, und leider haben sich alle unsere Befürchtungen bestätigt: Das isolierte ökonomische Denken setzt sich immer stärker durch in den Spitälern, der wirtschaftliche und zeitliche Druck auf das Spitalpersonal nimmt $\mathrm{zu}$, die versprochene Transparenz ist nach wie vor nicht vorhanden. Kostendämpfung gibt es keine, dafür Mengenausweitung und für die ärztliche Weiterbildung gibt es nach wie vor keine schweizweit akzeptierte Lösung. Die Akademie Menschenmedizin macht sich zur Aufgabe, diese Entwicklungen zu hinterfragen, öffentlich zu diskutieren sowie Thesen und Forderungen zu formulieren.
Noch gibt es zu wenige Engagierte, die sich aktiv wehren.

AHC: Ich bin empört über das viele Schweigen. Viele spüren das Unbehagen, doch sie funktionieren weiter passiv im System. Es muss doch noch mehr Leute geben, die den Freiraum und den Mut haben, sich gegen ungute Entwicklungen zu wehren.

Was tut die Akademie Menschenmedizin, um diese Unzufriedenen zu erreichen?

$\mathrm{CH}$ : Sie kann das unterirdische Unbehagen aufnehmen, das verbreitet im Gesundheitswesen spürbar ist, und es in produktive Bahnen leiten. Unbehagen ist der Trigger, um etwas zu unternehmen, aber dann braucht es lebbare Alternativen, eine Vision und eine erkenntnistheoretische Begründung, um das Wünschbare und das Machbare zusammenzuführen: Dort ist die Alternative, dorthin kann es gehen, damit sich Menschen wieder gemeint fühlen. Das braucht es auch, damit auf der professionellen Seite die intrinsische Motivation nicht verloren geht und Menschen wieder oder weiterhin mit Begeisterung ihren Beruf ausüben können.

AHC: Es gibt übrigens viele Möglichkeiten, bei der Akademie Menschenmedizin mitzumachen: Neben dem transdisziplinären Beirat haben wir eine Online-Interessengemeinschaft, wo sich Gleichgesinnte und Interessierte einbringen und austauschen können. Regelmässig finden politische Veranstaltungen statt, und für informelle Kontakte und Gespräche gibt es monatlich die Treff-Bar im Fischstübli in Erlenbach ZH.

Ende August findet das zweite Symposium Menschenmedizin zum Thema «Zeit» statt. Wie sind Sie auf dieses Thema gekommen?

AHC: Wir wollen die Stimmen der Jungen vom letzten Symposium aufnehmen. Es hat sich gezeigt, dass das Thema Zeit und Zeitnot die jungen Berufsleute im Gesundheitswesen besonders stark beschäftigt. $\mathrm{CH}$ : Wenn der Leitgedanke nur Effizienzsteigerung, also Rationalisieren ist, dann rationieren wir die Zeit. Es fragt sich, ob das der richtige Ort ist, um zu rationieren. Persönlich meine ich klar: nein. Auch dieses Thema wird am Symposium diskutiert werden.

\section{Symposium 2014 Zeit - Mensch - Medizin} Zeit haben - Zeitnot - den richtigen Zeitpunkt finden: Das Thema «Zeit», ein vielschichtiges und enorm wichtiges Thema für das Gesundheitswesen, wird von namhaften Referenten und Künstlerinnen aus unterschiedlichen Blickwinkeln beleuchtet.

Donnerstag, 28. August 2014, 9.00-17.00 Uhr Kunsthaus Zürich, Vortragssaal

Weitere Informationen und Anmeldung: www.menschenmedizin.ch Postadresse: Akademie Menschenmedizin, Husertalstrasse 9, 8925 Ebertswil 